\title{
Intermediation and Investment Incentives
}

P. Belleflamme and M. Peitz

Discussion Paper 2006-48

Département des Sciences Économiques

de l'Université catholique de Louvain 
CORE DISCUSSION PAPER 2006/94

\title{
Intermediation and Investment Incentives*
}

\author{
Paul Belleflamme ${ }^{\dagger}$ \\ Université Catholique de Louvain \\ Martin Peitz \\ International University in Germany and University of Mannheim
}

First version: April 2006. This version: September 2006

\begin{abstract}
We analyze whether and how the fact that products are not sold on free, public, platforms but on competing for-profit platforms affects sellers' investment incentives. Investments in cost reduction, quality, or marketing measures are here the joint and coordinated efforts by sellers. We show that, in general, for-profit intermediation is not neutral to such investment incentives. As for-profit intermediaries reduce the rents that are available in the market, one might suspect that sellers have weaker investment incentives with competing for-profit platforms. However, this is not necessarily the case. The reason is that investment incentives affect the size of the network effects and thus competition between intermediaries. In particular, we show that whether for-profit intermediation raises or lowers investment incentives depends on which side of the market singlehomes.
\end{abstract}

Keywords: Two-Sided Markets, Network Effects, Intermediation, Investment Incentives

JEL-Classification: L10, D40

${ }^{*}$ We would like to thank Jean Gabszewicz and Xavier Wauthy for helpful comments. Martin Peitz acknowledges financial support from the Deutsche Forschungsgemeinschaft (SFB TR 15).

${ }^{\dagger}$ CORE and IAG-Louvain School of Management, Université Catholique de Louvain, 34 Voie du Roman Pays, B-1348 Louvain la Neuve, Belgium, belleflamme@core.ucl.ac.be.

${ }^{\ddagger}$ International University in Germany, Campus 3, 76646 Bruchsal, Germany, Martin.Peitz@i-u.de 


\section{Introduction}

An important issue in the analysis of markets is about the strength of investment incentives that markets provide. While this is a question of high importance, little is known about the influence of market microstructure or trading environment on the investment incentives of the producers of goods and services that are traded via strategic for-profit platforms. We observe indeed that most consumer products are not sold directly but via intermediaries. These intermediaries may take various forms. Retailers such as Walmart may rent shelf space to producers, shopping mall developers rent stores to producers, trade fairs rent booths to exhibitors, internet retailers list products in their virtual shop. The users of these trading platforms may or may not pay for access and usage of the platform. Similarly, software platforms grant licences to software developers and charge users for access.

With the rise of $\mathrm{B} 2 \mathrm{~B}$ and $\mathrm{B} 2 \mathrm{C}$ commerce, this question has become even more relevant. The intermediaries may become active in different ways. They may fix bid and ask prices and therefore alleviate search inefficiencies, which arise e.g. under random matching. The presence of a dealer-intermediary can then be seen as an implicit screening device between seller and buyer types (see e.g. Gehrig, 1993, and Spulber, 2003). In many markets, however, intermediaries only charge for usage and access of a trading platform. Here, search inefficiencies may be so pronounced that sellers cannot circumvent a platform. This is clearly the case if the platform provides part of a system that complements the product provided by the seller. A good example for this is the video game industry (and other software industries) in which game developers write their applications for particular game platforms. In this case, a video game platform aggregates demand and balances the two sides of the market through the use of price instruments (as in the literature on two-sided markets, see e.g. Rochet and Tirole, 2003, and Armstrong, 2006). In such an industry, we can abstract from any search efficiencies but rather consider indirect network effects that arise due to group size.

The particular question we address in this paper is how seller investment incentives are affected by the presence of competing platforms. To this end, we present a stylized model of two competing platforms. Participants on both sides of the market choose which platform to visit; we contrast different scenarios according to whether buyers and/or sellers are allowed to trade via both platforms (i.e., to multihome) or are restricted to use a single platform (i.e., to singlehome). We capture size effects in the form of variety seeking buyers who have a downward-sloping demand function for each product that is available. Our benchmark is 
a market in which buyers and sellers interact through two public platforms, whose access is free of charge. To address the market failure due to (imperfectly) competing intermediaries, we compare investment incentives with these free platforms to those with two competing for-profit platforms.

Investments in cost reduction, quality, or marketing measures are here the joint and coordinated efforts by sellers (in the form of horizontal agreements). In particular, they may take the form of R\&D joint ventures. Clearly, the presence of intermediaries reduces the rents that are available in the market. Therefore, one might suspect that sellers have weaker investment incentives with intermediated trade. Another reason is that with two platforms who share the market, indirect network effects are less pronounced, which also tends to lead to weaker investment incentives with intermediated trade.

However, this is not necessarily the case. The reason is that investment incentives affect the size of the network effects and thus competition between intermediaries. Our main result is that incentives to innovate are stronger with strategic platforms than with free public platforms depending on which side of the market singlehomes and on the nature of the innovation. More precisely, we show that (i) when both sides singlehome, trade via strategic platforms raises incentives to innovate in cost-reduction and in quality, but lowers incentives to innovate in marketing measures; (ii) when sellers singlehome and buyers can multihome, trade via strategic platforms leads to stronger incentives to innovate whatever the nature of the innovation; (iii) when buyers singlehome and sellers can multihome, the exact opposite prevails (trade via strategic platforms reduces incentives to innovate).

Related literature. Our paper contributes to the burgeoning literature on two-sided markets. Compatible with this literature is the view that intermediaries possess property rights on a platform and thus can make profits from charging access or usage fees on both sides of the market. Seminal contributions in this literature are Caillaud and Jullien (2003), Rochet and Tirole (2003, 2006), and Armstrong (2006). Our set-up of the two-sided market borrows from Armstrong's models with singlehoming and with competitive bottlenecks where two competing intermediaries set access prices on both sides of the market. In this model, we provide a micro foundation of seller profits and consumer utilities and analyze sellers' investment incentives in comparison to a model in which all sellers and buyers trade via free platforms.

An interesting analysis of seller investment incentives in the context of media is provided by Stennek (2006). He considers a model in which a monopoly seller of content can sign an 
exclusive contract with a single platform or multihome and offer its content on both platforms. Seller and platform play an extensive form bargaining game with alternating offers. Since under exclusivity the excluded party is at a disadvantage, the contracting parties inflict a negative externality on the excluded party. He shows that exclusive contracts are chosen in equilibrium if the joint profit of the contracting parties is higher under exclusivity than under non-exclusivity. Exclusive distribution may give stronger incentives to develop higher quality (and may force the excluded competitor to price more aggressively). Government interventions that ban exclusivity may then harm consumers. While Stennek highlights the effect of exclusivity on seller incentives, our paper sheds some light on seller incentives in a for-profit compared to a non-profit environment with respect to the platforms' objectives.

Our paper also contributes to the micro-market structure and intermediation literature more generally. Here, an alternative branch of literature has taken the view that intermediated trade (by dealers who set bid and ask prices) may avoid inefficiencies that arise in random matching environments. In this setting, the coexistence of matching and dealer market leads to a self-selection of types (see Gehrig, 1993). ${ }^{1}$ In such an environment, Spulber (2003) analyzes sellers' (and buyers') investment incentives. He shows that the introduction of a dealer market in a decentralized matching market leads to stronger investment incentives. Our analysis can be seen as complementary to the work by Spulber (2003) because we abstract from search inefficiencies and rather focus on market size externalities.

Our paper also contributes to the literature on $R \& D$ joint ventures and $R \& D$ agreements (see Kamien, Muller, and Zang, 1992, and the literature that builds on this). These joint ventures or agreements imply certain investment decisions by the participating firms and innovations are shared among the firms. In particular, research joint ventures pool resources within a common functional area to overcome capacity constraints in research activities, pool resources across functions areas, or develop new products or processes in parallel and then share the innovation. The contribution of this paper then is to show that the incentives to form such joint ventures or agreements and the particular policy that is chosen by the firms depend on the underlying market microstructure. In particular, it may be affected by the need to obtain access to one of the competing platforms. Similarly, concerning marketing alliances at the producer level, the intensity of joint marketing activities depend on the underlying market microstructure. ${ }^{2}$

\footnotetext{
${ }^{1}$ For a presentation of the Gehrig model, see Spulber (1999). For an extended analysis, see Rust (2003).

${ }^{2}$ As another example, we can think of the investment by music labels against piracy, which is coordinated by the RIAA (and thus constitutes a joint investment). These incentives are affected by the need to have
} 
In a recent empirical paper, Boudreau (2006) investigates the effect of the degree of openness of the platform on seller incentives in the computer industry. He finds that restricted access and some control over the platform led to more investments in innovation than highly open strategies by platforms. A potential reason for these findings, as has been recognized by Boudreau (2006, p.2 ), are strategic effects: "In that the opening of a system will also surely affect the 'within-system' competition (and perhaps even between system strategic interactions), suppliers' strategic incentives to make investments in innovation might also be affected." This paper presents a formal framework to address the issue of competition between platforms.

The rest of the paper is organized as follows. In Section 2, we set up the general model. We then analyze three particular versions of the model: in Section 3, we assume that both buyers and sellers singlehome; in Section 4, only buyers singlehome while sellers are allowed to multihome; in Section 5, the opposite prevails as sellers singlehome while buyers are allowed to multihome. In Section 6, we provide a microfoundation for the generic surplus functions used in the previous sections; thereby we examine the seller incentives to innovate in costreduction, in quality improvement, and in price discrimination. We conclude and discuss possible extensions in Section 7.

\section{The model}

There are two sides of the market, the buyer side and the seller side. Suppose that each side is of mass 1. Buyers and sellers can interact on two platforms, 1 and 2, which are assumed to be located at the extreme points of the unit interval. Buyers are of two (exogenously given) types: a mass $\lambda_{b}$ of the buyers can multihome (i.e., they can trade on both platforms), while the complementary mass $\left(1-\lambda_{b}\right)$ can only singlehome (i.e., they can trade on at most one platform), with $0 \leq \lambda_{b} \leq 1$; each subset of buyers is assumed to be uniformly distributed on the unit interval. The seller side is represented in a similar way: a mass $0 \leq \lambda_{s} \leq 1$ of the sellers can multihome, while a mass $\left(1-\lambda_{s}\right)$ can only singlehome. Buyers and sellers are assumed to incur an opportunity cost of visiting a platform which increases linearly in distance at rates $\tau_{b}$ and $\tau_{s}$, respectively.

In this setting, we analyze the ex ante investment incentives of sellers, supposing that

music distributed by a platform such as iTunes. Similarly, the lobbying efforts by industry associations, e.g. in favor of minimum quality standards, can be interpreted as investments on the supplier side. 
sellers can coordinate their investment decisions. We will compare the sellers' incentives to innovate under two different organizations of the trading platforms: intermediated trade (in which platforms are run by strategic intermediaries) and non-intermediated trade (in which platforms are public).

In the case of intermediated trade, the timing of the game is as follows:

Stage 1 Sellers make investment decision $y$ (as a coordinated effort).

Stage 2 Intermediaries simultaneously set membership fees $M_{s}^{i}, M_{b}^{i}$ on the two sides of the market.

Stage 3 Sellers and buyers decide which intermediary to visit.

Stage 4 Sellers set their strategic variable simultaneously.

Stage 5 Buyers make purchasing decisions.

Regarding Stage 4, we assume that the sellers' pricing decisions are independent, so that we do not need to make particular assumptions on the timing decision at this pricing stage (we can think of sellers producing perfectly differentiated varieties). This assumption simplifies the analysis and allows us to focus on comparing investment decisions under intermediated and non-intermediated trade. Moreover, as we show in the appendix, our results are robust to introducing competition among sellers as they continue to hold in a richer setting where competition prevails among sellers. Regarding Stage 5, we assume that a buyer at platform $i$ buys one unit from each seller trading on this platform. We will provide below a number of micro models of buyers-seller relationships and analyze particular investment decisions in such settings. In particular, we consider sellers with independent and downward sloping demand who engage in cost-reducing $\mathrm{R} \& \mathrm{D}$, who invest in quality improvements, or who invest in technologies which allow them to price discriminate between consumers. As for now, we use a reduced-form representation of buyer-seller interaction. Buyer and seller surplus gross of any opportunity cost of visiting a platform are simply computed as

$$
v_{s}^{i}=n_{b}^{i} \pi-M_{s}^{i} \text { and } v_{b}^{i}=n_{s}^{i} u-M_{b}^{i},
$$

where $\pi$ is the net gain from trade for each seller, $u$ is the net gain from trade for each buyer, $n_{b}^{i}$ (resp. $n_{s}^{i}$ ) is the number of buyers (resp. sellers) active on platform $i$, and $M_{b}^{i}$ and $M_{s}^{i}$ are the membership fees set by intermediary $i$. 
Using these functions, we can analyze the buyers' and sellers' platform choices at Stage 3. Consider first the buyer side. Let $b_{12}$ denote the buyer who is indifferent between visiting platform 1 and visiting platform 2 . That is, $b_{12}$ is defined by

$$
v_{b}^{1}-\tau_{b} b_{12}=v_{b}^{2}-\tau_{b}\left(1-b_{12}\right) \Longleftrightarrow b_{12}=\frac{1}{2}+\frac{v_{b}^{1}-v_{b}^{2}}{2 \tau_{b}} .
$$

Similarly, let $b_{10}$ (resp. $b_{20}$ ) denote the buyer who is indifferent between visiting platform 1 (resp. 2) and not visiting any platform (thereby getting a utility of zero):

$$
\begin{aligned}
v_{b}^{1}-\tau_{b} b_{10} & =0 \Longleftrightarrow b_{10}=\frac{v_{b}^{1}}{\tau_{b}}, \\
v_{b}^{2}-\tau_{b}\left(1-b_{20}\right) & =0 \Longleftrightarrow b_{20}=1-\frac{v_{b}^{2}}{\tau_{b}} .
\end{aligned}
$$

We proceed in the same way on the seller side by defining the indifferent sellers as:

$$
s_{12}=\frac{1}{2}+\frac{v_{s}^{1}-v_{s}^{2}}{2 \tau_{s}}, s_{10}=\frac{v_{s}^{1}}{\tau_{s}}, \text { and } s_{20}=1-\frac{v_{s}^{2}}{\tau_{s}} .
$$

In what follows, we will assume that participation is sufficiently attractive so that all buyers and sellers participate in the market. More precisely, we require (i) $0<b_{20}<b_{12}<$ $b_{10}<1$ and (ii) $0<s_{20}<s_{12}<s_{10}<1$. Necessary and sufficient conditions are

$$
\begin{aligned}
& v_{b}^{1}, v_{b}^{2}>0 \text { and } \max \left\{v_{b}^{1}, v_{b}^{2}\right\}<\tau_{b}<v_{b}^{1}+v_{b}^{2} \\
& v_{s}^{1}, v_{s}^{2}>0 \text { and } \max \left\{v_{s}^{1}, v_{s}^{2}\right\}<\tau_{s}<v_{s}^{1}+v_{s}^{2}
\end{aligned}
$$

Under condition (1), the buyers who singlehome are divided into two groups: those located between 0 and $b_{12}$ visit platform 1 and those located between $b_{12}$ and 1 visit platform 2 . As for the buyers who can multihome, they are divided into three groups: those located between 0 and $b_{20}$ visit platform 1 only, those located between $b_{20}$ and $b_{10}$ visit both platforms, and those located between $b_{10}$ and 1 visit platform 2 only. Recalling that the proportions of multihomers and singlehomers are, respectively, $\lambda_{b}$ and $1-\lambda_{b}$, we compute the number of buyers visiting each platform as $n_{b}^{1}=\lambda_{b} b_{10}+\left(1-\lambda_{b}\right) b_{12}$ and $n_{b}^{2}=\lambda_{b}\left(1-b_{20}\right)+\left(1-\lambda_{b}\right)\left(1-b_{12}\right)$, or

$$
\begin{aligned}
& n_{b}^{1}=\lambda_{b} \frac{v_{b}^{1}}{\tau_{b}}+\left(1-\lambda_{b}\right)\left(\frac{1}{2}+\frac{v_{b}^{1}-v_{b}^{2}}{2 \tau_{b}}\right), \\
& n_{b}^{2}=\lambda_{b} \frac{v_{b}^{2}}{\tau_{b}}+\left(1-\lambda_{b}\right)\left(\frac{1}{2}+\frac{v_{b}^{2}-v_{b}^{1}}{2 \tau_{b}}\right) .
\end{aligned}
$$


Applying the same reasoning and assuming that condition (2) is met, we compute the number of sellers visiting each platform as:

$$
\begin{aligned}
& n_{s}^{1}=\lambda_{s} \frac{v_{s}^{1}}{\tau_{s}}+\left(1-\lambda_{s}\right)\left(\frac{1}{2}+\frac{v_{s}^{1}-v_{s}^{2}}{2 \tau_{s}}\right), \\
& n_{s}^{2}=\lambda_{s} \frac{v_{s}^{2}}{\tau_{s}}+\left(1-\lambda_{s}\right)\left(\frac{1}{2}+\frac{v_{s}^{2}-v_{s}^{1}}{2 \tau_{s}}\right) .
\end{aligned}
$$

In contrast, in the case of non-intermediated trade, the game is simplified as the strategic intermediaries disappear. Buyers and sellers interact through public platforms whose access is assumed to be free of charge. Therefore, the timing is as follows: sellers first decide on the investment and then set prices; finally, buyers make their purchasing decision.

We now analyze the following three scenarios: (i) both sides of the market singlehome, (ii) all sellers multihome and all buyers singlehome, and (iii) all sellers singlehome and all buyers multihome. ${ }^{3}$ For each scenario, we contrast the sellers' incentives to innovate under intermediated and non-intermediated trade.

\section{Two-sided single homing}

We suppose here that both sides of the market singlehome. ${ }^{4}$ Singlehoming environments in the real world can be motivated by indivisibilities and limited resources, or by contractual restrictions. The former applies to certain real-world market places where buyers and sellers can physically locate in only one of them (flea and farmer markets come to mind). For the latter, we find more examples. For instance, taxi companies in Germany sign exclusive contracts with taxi call centers. There also appears to be little multihoming on the consumer side. Similarly, some employment agencies for temporary work can be characterized by singlehoming on both sides of the market. Video game platform can also be approximated by singlehoming. Admittedly, some gamers have more than one platforms and some game developers develop the same game for different platforms. However, the majority of games are released exclusively for one platform and a discrete choice set-up seems to be a reasonable

\footnotetext{
${ }^{3}$ Our model is not tractable to analyse the remaining case where both sides multihome. No interior solution obtains in this setting. Anyway, this case is not expected to be very common: if one side of the market multihomes, it provides full access to the other side of the market, which is therefore at least as well off singlehoming.

${ }^{4}$ The analysis draws here on the singlehoming model by Armstrong (2006).
} 
approximation for consumers. ${ }^{5}$ Also, in certain markets for magazines, it has been claimed that the market for certain specialized magazines, where the magazine serves as the platform and advertisers and readers constitute the two sides of the market, can be described as a market on which both sides singlehome (see Kaiser and Wright, 2006). In addition, many media markets have the property that content is exclusive. If content providers sell their content to consumers while the media platform only charges for the services it offers, we are also in a situation where both sides singlehome (presuming that consumers do not multihome).

To model such a situation, we set both $\lambda_{b}$ and $\lambda_{s}$ to zero. Expressions (3) to (6) therefore rewrite as the standard Hotelling specifications,

$$
n_{b}^{i}=\frac{1}{2}+\frac{v_{b}^{i}-v_{b}^{j}}{2 \tau_{b}} \text { and } n_{s}^{i}=\frac{1}{2}+\frac{v_{s}^{i}-v_{s}^{j}}{2 \tau_{s}} .
$$

Using the expressions for buyer and seller surplus and the facts that $n_{b}^{j}=1-n_{b}^{i}$ and $n_{s}^{j}=1-n_{s}^{i}$, we obtain the following expressions for the numbers of buyers and sellers at the two platforms:

$$
\begin{aligned}
& n_{b}^{i}=\frac{1}{2}+\frac{\left(2 n_{s}^{i}-1\right) u-\left(M_{b}^{i}-M_{b}^{j}\right)}{2 \tau_{b}}, \\
& n_{s}^{i}=\frac{1}{2}+\frac{\left(2 n_{b}^{i}-1\right) \pi-\left(M_{s}^{i}-M_{s}^{j}\right)}{2 \tau_{s}} .
\end{aligned}
$$

This shows that for given membership fees of buyers, an additional seller attracts $u / \tau_{b}$ additional buyers. If the gains from trade $u$ and $\pi$ are large relative to opportunity costs, two intermediaries cannot be active because indirect network effects are too strong. To exclude this possibility, we assume that $4 \tau_{s} \tau_{b}>(u+\pi)^{2}{ }^{6}$ We can then solve the above implicit

\footnotetext{
${ }^{5}$ Clements and Ohashi (2005) report that only $17 \%$ of titles in their sample was available on multiple platforms. They estimate a structural discrete choice model so that consumers are assumed to singlehome. Other authors have argued that multihoming on the developer side is common. E.g., Evans and Schmalensee (2005) report that in 2003 Electronic Arts, a leading game developer, developed for the Nintendo, Microsoft, and Sony platforms. However, to talk about multihoming, it matters more whether particular games are sold exclusively or on various platforms; whether a multi-product firm sells different products on different platforms appears to be less relevant in the present context. A business rationale may simply be to hedge the risk to develop for the wrong platform.

${ }^{6}$ This is the necessary and sufficient condition for an equilibrium to exist in which both intermediaries are active.
} 
expressions for the number of buyers and sellers to obtain the following formulas:

$$
\begin{aligned}
& n_{b}^{i}=\frac{1}{2}+\frac{u\left(M_{s}^{j}-M_{s}^{i}\right)+\tau_{s}\left(M_{b}^{j}-M_{b}^{i}\right)}{2\left(\tau_{b} \tau_{s}-u \pi\right)}, \\
& n_{s}^{i}=\frac{1}{2}+\frac{\pi\left(M_{b}^{j}-M_{b}^{i}\right)+\tau_{b}\left(M_{s}^{j}-M_{s}^{i}\right)}{2\left(\tau_{b} \tau_{s}-u \pi\right)} .
\end{aligned}
$$

The number of buyers at one platform is not only decreasing in the membership fee for buyers on this platform but also, due to indirect network effects, in the membership fee for sellers.

Let us now turn to the first two stages of the game.

\subsection{Pricing by intermediaries}

Assuming that the intermediary's cost per buyer is $C_{b}$ and per seller is $C_{s}$, we can write platform $i$ 's profit as

$$
\begin{aligned}
\Pi^{i}= & \left(M_{s}^{i}-C_{s}\right)\left(\frac{1}{2}+\frac{\pi\left(M_{b}^{j}-M_{b}^{i}\right)+\tau_{b}\left(M_{s}^{j}-M_{s}^{i}\right)}{2\left(\tau_{b} \tau_{s}-u \pi\right)}\right) \\
& +\left(M_{s}^{i}-C_{s}\right)\left(\frac{1}{2}+\frac{u\left(M_{s}^{j}-M_{s}^{i}\right)+\tau_{s}\left(M_{b}^{j}-M_{b}^{i}\right)}{2\left(\tau_{b} \tau_{s}-u \pi\right)}\right) .
\end{aligned}
$$

The two intermediaries simultaneously choose membership fees on both sides of the market. First-order conditions of profit maximization in a symmetric equilibrium, i.e. $M_{s}^{1}=$ $M_{s}^{2} \equiv M_{s}$ and $M_{b}^{1}=M_{b}^{2} \equiv M_{b}$, can be written as

$$
\begin{aligned}
& M_{s}=C_{s}+\tau_{s}-\frac{u}{\tau_{b}}\left(\pi+M_{b}-C_{b}\right), \\
& M_{b}=C_{b}+\tau_{b}-\frac{\pi}{\tau_{s}}\left(u+M_{s}-C_{s}\right) .
\end{aligned}
$$

Equilibrium prices are equal to marginal costs plus the product differentiation term as in the standard Hotelling model, adjusted downward by the term $\frac{u}{\tau_{b}}\left(\pi+M_{b}-C_{b}\right)$. Recall that each additional seller attracts $u / \tau_{b}$ additional buyers. These additional buyers allow the intermediary to extract $\pi$ per seller without affecting the sellers' surplus. In addition, each of the additional $u / \tau_{b}$ buyers gives a profit of $M_{b}-C_{b}$ to the seller. Thus $\frac{u}{\tau_{b}}\left(\pi+M_{b}-C_{b}\right)$ represents the value of an additional buyer to the intermediary. The higher this value, the more aggressive the price setting among intermediaries. 
Solving for the Nash equilibrium membership fees, one finds ${ }^{7}$

$$
\begin{aligned}
& M_{s}^{*}=C_{s}+\tau_{s}-u, \\
& M_{b}^{*}=C_{b}+\tau_{b}-\pi .
\end{aligned}
$$

It follows that at equilibrium, $n_{b}^{1 *}=n_{b}^{2 *}=1 / 2$ and $n_{s}^{1 *}=n_{s}^{2 *}=1 / 2$, so that the equilibrium net surplus of sellers and buyers (gross of transportation cost) are equal to: ${ }^{8}$

$$
\begin{aligned}
& v_{s}^{*}=\frac{1}{2} \pi+u-\left(C_{s}+\tau_{s}\right), \\
& v_{b}^{*}=\frac{1}{2} u+\pi-\left(C_{b}+\tau_{b}\right) .
\end{aligned}
$$

We observe that $v_{s}$ and $v_{b}$ are increasing in the net gain of the other side and, to a lesser extent, also in the net gain of the own side. ${ }^{9}$

The intermediaries' equilibrium profits are

$$
\Pi^{i *}=\frac{1}{2}\left(\tau_{s}-u\right)+\frac{1}{2}\left(\tau_{b}-\pi\right)=\frac{1}{2}\left(\tau_{s}+\tau_{b}\right)-\frac{1}{2}(u+\pi) .
$$

Note that only the joint net gain from trade by buyers and sellers determines the intermediary's profit. Thus, the distribution of net gains among sellers and buyers does not affect the intermediaries profit. Furthermore, this profit is decreasing in $(u+\pi)$; i.e., in markets in which gains from trade are high the intermediary's profits are low. This result may seem counterintuitive but can be explained as follows. Net gains $u$ and $\pi$ determine the strength of network effects in the industry. If $u+\pi$ is large this means that additional buyers and sellers are very valuable for intermediaries. Therefore, they compete more aggressively in the market place. If network effects were too strong only one intermediary would be viable. We restrict attention to situations in which two intermediaries are viable so that $\Pi^{i *}>0$. This is the case under the assumption we made above, according to which $4 \tau_{s} \tau_{b}>(u+\pi)^{2}$, which implies that platform are sufficiently differentiated.

\footnotetext{
${ }^{7}$ As indicated above, the assumption that $4 \tau_{b} \tau_{s}>(\pi+u)^{2}$ makes sure that the second-order conditions are satisfied.

${ }^{8}$ We still need to check that conditions (1) and (2) are met. This is so provided that $\frac{1}{2}\left(\frac{1}{2} u+\pi-C_{b}\right)<$ $\tau_{b}<\frac{2}{3}\left(\frac{1}{2} u+\pi-C_{b}\right)$ and $\frac{1}{2}\left(\frac{1}{2} \pi+u-C_{s}\right)<\tau_{s}<\frac{2}{3}\left(\frac{1}{2} \pi+u-C_{s}\right)$.

${ }^{9}$ Note that, in a bargaining environment, this implies that sellers would rather benefit from less bargaining power in a buyer-seller relationship. This may sound counterintuitive. Clearly, in each buyer-seller relationship a seller benefits from an increase in his bargaining power, everything else equal. However, in the intermediated market the shift in bargaining power affects membership fees. Taking these effects into account, a seller would like to be weak in the bargaining process.
} 


\subsection{Interaction through public platforms}

To model non-intermediated trade, we consider two public platforms located at the extremes of the unit interval (that is, they have the same locations as the strategic platforms in the alternative environment). In this case, each seller has access to half of the unit mass of consumers and derives net surplus (but gross of transport costs) equal to $\pi / 2$ (supposing that access to each public platform is free). ${ }^{10}$

\subsection{Seller incentives to innovate}

To evaluate the sellers' incentives to innovate, suppose that sellers jointly determine their efforts in some R\&D or marketing activity that affects some parameter $y$ that enters the profit function of each firm. We can then write maximal profit (in some examples equilibrium profits) as a function $\pi(y) .{ }^{11}$ Note that a change in $y$ typically affects user surplus as well. Therefore, we write surplus as a function of $y$, i.e. $u(y)$. The incentives to innovate are then determined by the effect of $y$ on $\pi$ and $u$.

How much is a seller willing to pay to acquire this innovation? Consider an innovation from $y$ to $y^{\prime}$. In the non-intermediated market (where buyers and sellers trade on two public platforms), each seller is willing to pay up to the increase in its profit for an innovation from $y$ to $y^{\prime}$ :

$$
\Delta^{n} \equiv \frac{1}{2}\left[\pi\left(y^{\prime}\right)-\pi(y)\right]
$$

In the intermediated market (where trade occurs via strategic platforms), sellers are willing to pay up to the increase in their net surpluses: $\Delta^{m} \equiv v_{s}\left(y^{\prime}\right)-v_{s}(y)$. From (9), we obtain

$$
\Delta^{m}=\frac{1}{2}\left[\pi\left(y^{\prime}\right)-\pi(y)\right]+\left[u\left(y^{\prime}\right)-u(y)\right]
$$

Comparing the expressions for $\Delta^{n}$ and $\Delta^{m}$, we have the following simple result.

Proposition 1 In the two-sided singlehoming model, strategic trading platforms give stronger incentives for sellers to innovate if the buyers' surplus increases, i.e.

$$
\Delta^{m}>\Delta^{n} \Longleftrightarrow u\left(y^{\prime}\right)-u(y)>0 .
$$

\footnotetext{
${ }^{10}$ Note that in a bargaining environment, in each buyer-seller relationship, a seller benefits from an increase in his bargaining power and thus would like to be strong in the bargaining process.

${ }^{11}$ We provide micro foundations for this profit function in Section 6 .
} 
To better understand the previous conditions, we will, in Section 6 , have a closer look at the microstructure of the buyer-seller relationship and the nature of seller investments. Note that since $n_{s}^{i}=1 / 2$ under both types of platform organization, the ranking of per-seller incentives implies the same order of total investment (i.e., the sum of sellers' willingness to pay for the innovation).

\section{Competitive bottlenecks when sellers multihome}

We suppose here that all buyers can only singlehome (so that $\lambda_{b}=0$ ) while all sellers have the possibility to multihome (so that $\lambda_{s}=1$ ). As noted by Evans (2003), personal computers constitute a typical example of this situation: end-users (i.e., buyers) singlehome (they almost always use a single operating system), while application developers (i.e., sellers) do multihome. ${ }^{12}$

Expressions (3) to (6) now rewrite shortly as

$$
n_{b}^{i}=\frac{1}{2}+\frac{v_{b}^{i}-v_{b}^{j}}{2 \tau_{b}} \text { and } n_{s}^{i}=\frac{v_{s}^{i}}{\tau_{s}}
$$

or equivalently, using the expressions for buyer and seller surplus, as

$$
n_{b}^{i}=\frac{1}{2}+\frac{u\left(n_{s}^{i}-n_{s}^{j}\right)-\left(M_{b}^{i}-M_{b}^{j}\right)}{2 \tau_{b}} \text { and } n_{s}^{i}=\frac{n_{b}^{i} \pi-M_{s}^{i}}{\tau_{s}} .
$$

Solving this system of four equations in four unknowns, we get

$$
\begin{aligned}
n_{b}^{i} & =\frac{1}{2}+\frac{u\left(M_{s}^{j}-M_{s}^{i}\right)+\tau_{s}\left(M_{b}^{j}-M_{b}^{i}\right)}{2\left(\tau_{b} \tau_{s}-u \pi\right)} \\
n_{s}^{i} & =\frac{\pi}{\tau_{s}}\left(\frac{1}{2}+\frac{u\left(M_{s}^{j}-M_{s}^{i}\right)+\tau_{s}\left(M_{b}^{j}-M_{b}^{i}\right)}{2\left(\tau_{b} \tau_{s}-u \pi\right)}\right)-\frac{M_{s}^{i}}{\tau_{s}} .
\end{aligned}
$$

Comparing expressions (7) and (11) and supposing that membership fees do not change, we observe that the number of buyers is the same whether sellers are allowed to multihome or not. As for sellers, the comparison of expressions (8) and (12) reveals that (still supposing that membership fees do not change) the number of sellers at platform $i$ is larger when sellers are allowed to multihome if $\pi>M_{s}^{i}+M_{s}^{j}+\tau_{s}$.

Let us now turn to the first two stages of the game.

\footnotetext{
${ }^{12}$ See Lerner (2002) for data about the number of developers that develop for various operating systems.
} 


\subsection{Pricing by intermediaries}

Each platform $i$ solves the problem $\max _{M_{b}^{i}, M_{s}^{i}} \Pi^{i}$ where

$$
\Pi^{i}=\left(M_{b}^{i}-C_{b}\right) n_{b}^{i}\left(M_{b}^{i}, M_{b}^{j}, M_{s}^{i}, M_{s}^{j}\right)+\left(M_{s}^{i}-C_{s}\right) n_{s}^{i}\left(M_{b}^{i}, M_{b}^{j}, M_{s}^{i}, M_{s}^{j}\right) .
$$

Firm's best responses are implicitly defined by the first-order conditions which can be expressed as

$$
\begin{aligned}
M_{b}^{1} & =\frac{-(u+\pi) M_{s}^{1}-2 \tau_{s} M_{b}^{1}+u M_{s}^{2}+\tau_{s} M_{b}^{2}-\pi\left(u-C_{s}\right)+\tau_{s}\left(\tau_{b}+C_{b}\right)}{2 \tau_{s}} \\
M_{s}^{1} & =\frac{(u+\pi) \tau_{s} M_{b}^{1}-u \pi M_{s}^{2}-\pi \tau_{s} M_{b}^{2}-\left(\pi+2 C_{s}\right) \tau_{b} \tau_{s}+\pi u\left(\pi+C_{s}\right)-u \tau_{s} C_{b}}{2\left(u \pi-2 \tau_{b} \tau_{s}\right)} .
\end{aligned}
$$

Second-order conditions require that $8 \tau_{b} \tau_{s}>\pi^{2}+u^{2}+6 \pi u$. Equilibrium prices are

$$
\begin{aligned}
& M_{b}^{1 *}=M_{b}^{2 *}=\tau_{b}+C_{b}-\frac{\pi}{4 \tau_{s}}\left(3 u+\pi-2 C_{s}\right), \\
& M_{s}^{1 *}=M_{s}^{2 *}=\frac{1}{2} C_{s}+\frac{1}{4}(\pi-u) .
\end{aligned}
$$

On the seller side, platforms have monopoly power. If the intermediary focused only on sellers, he would charge a monopoly price equal to $C_{s} / 2+\pi / 4$ (assuming that each seller would have access to half of the buyers and, therefore, would have a gross willingness to pay equal to $\pi / 2$ ). We observe that this price is adjusted downward by $u / 4$ when the indirect network effects are taken into account (and remains positive as long as $\pi+2 C_{s}>u$ ). Similarly, on the buyer side, platforms charge the Hotelling price $\tau_{b}+C_{b}$ less a term that depends on the size of the indirect network effects.

It follows that at equilibrium,

$$
\begin{aligned}
& n_{b}^{1 *}=n_{b}^{2 *}=\frac{1}{2} \\
& n_{s}^{1 *}=n_{s}^{2 *}=\frac{1}{4 \tau_{s}}\left(u+\pi-2 C_{s}\right)
\end{aligned}
$$

Thus we must have $0<n_{s}^{i}<1 \Longleftrightarrow 2 C_{s}<u+\pi<2 C_{s}+4 \tau_{s}$ for obtaining an interior solution. ${ }^{13}$ Under this condition, the equilibrium net surplus of sellers and buyers (gross of transportation cost and for one platform) are equal to:

$$
\begin{aligned}
v_{s}^{*} & =\frac{1}{4}(u+\pi)-\frac{1}{2} C_{s}, \\
v_{b}^{*} & =\frac{1}{4 \tau_{s}}\left(u^{2}+4 \pi u+\pi^{2}-2(u+\pi) C_{s}\right)-\tau_{b}-C_{b} .
\end{aligned}
$$

\footnotetext{
${ }^{13}$ More precisely, conditions (1) and (2) rewrite here as: $K /\left(8 \tau_{s}\right)<\tau_{b}<K /\left(6 \tau_{s}\right)$ with $K \equiv u^{2}+4 \pi u+$ $\pi^{2}-2(u+\pi) C_{s}-4 \tau_{s} C_{b}$, and $\frac{1}{4}\left(u+\pi-2 C_{s}\right)<\tau_{s}<\frac{1}{2}\left(u+\pi-2 C_{s}\right)$.
} 
Note that $v_{s}^{*}$ is the per platform seller's surplus. Suppose that $n_{s}^{i *}>1 / 2$, i.e. some sellers multi-home and $v_{s}^{*}$ is the surplus earned by the sellers located between 0 and $1-\left(v_{s}^{*} / \tau_{s}\right)$, who choose to visit platform 1 only, and by the sellers located between $v_{s}^{*} / \tau_{s}$ and 1 , who choose to visit platform 2 only. The remaining sellers located between $1-\left(v_{s}^{*} / \tau_{s}\right)$ and $v_{s}^{*} / \tau_{s}$ multihome and, therefore, earn a surplus of $2 v_{s}^{*}$. (If on the other hand, $n_{s}^{i *}<1 / 2$, which is equivalent to $u+\pi<2 C_{s}+2 \tau_{s}$, sellers between $\left(u+\pi-2 C_{s}\right) /\left(4 \tau_{s}\right)$ and $1-\left(u+\pi-2 C_{s}\right) /\left(4 \tau_{s}\right)$ would not participate.) We observe that $v_{s}^{*}$ and $v_{b}^{*}$ are increasing in the net gain of the other side and in the net gain of the own side. The intermediaries' equilibrium profits are

$$
\Pi^{i *}=\frac{1}{16 \tau_{s}}\left(8 \tau_{b} \tau_{s}-\left(\pi^{2}+u^{2}+6 \pi u\right)+4 C_{s}^{2}\right)>0
$$

\subsection{Interaction through public platforms}

As in the previous section, consider two platforms located at the extremes of the unit interval (that is, they have the same locations as strategic platforms). Suppose access is free. Setting $M_{b}^{i}=M_{b}^{j}=M_{s}^{i}=M_{s}^{j}=0$ in expressions (11) and (12), we compute the numbers of buyers and sellers on each platform as

$$
n_{b}^{1}=n_{b}^{2}=\frac{1}{2} \text { and } n_{s}^{1}=n_{s}^{2}=\frac{1}{2 \tau_{s}} \pi
$$

It follows that the (per platform) net surplus of sellers and buyers are equal to

$$
v_{s}^{n}=\frac{1}{2} \pi \text { and } v_{b}^{n}=\frac{1}{2 \tau_{s}} \pi u
$$

Assuming that $\pi<2 \tau_{s}$, we have that the sellers located between $1-\pi /\left(2 \tau_{s}\right)$ and $\pi /\left(2 \tau_{s}\right)$ visit both public platforms, whereas the other sellers visit only the platform close to their location. As long as the equilibrium fee set by strategic platforms, $M_{s}^{i *}$, is positive, free public platforms attract more sellers than strategic platforms (which also means that more sellers multihome if trade is organized through free public platforms).

\subsection{Seller incentives to innovate}

As before, we compare the sellers' incentives to innovate under two different organizations of the trading platforms, a situation in which there are two strategic platforms and a situation in which there are two free public platforms. Using the same notation as in the previous section, with two strategic intermediaries, sellers are willing to pay up to the increase in their 
(equilibrium) net surpluses $\Delta^{m} \equiv v_{s}\left(y^{\prime}\right)-v_{s}(y)$. Here, we need to distinguish between the sellers who multihome and those who singlehome at equilibrium The net surplus is given by

$$
\Delta^{m}= \begin{cases}\frac{1}{2}\left(u\left(y^{\prime}\right)-u(y)+\pi\left(y^{\prime}\right)-\pi(y)\right) & \text { for multihoming sellers } \\ \frac{1}{4}\left(u\left(y^{\prime}\right)-u(y)+\pi\left(y^{\prime}\right)-\pi(y)\right) & \text { for singlehoming sellers }\end{cases}
$$

If trade takes place on two free public platforms, the increase in the sellers' net surpluses is

$$
\Delta^{n}= \begin{cases}\pi\left(y^{\prime}\right)-\pi(y) & \text { for multihoming sellers } \\ \frac{1}{2}\left(\pi\left(y^{\prime}\right)-\pi(y)\right) & \text { for singlehoming sellers }\end{cases}
$$

Comparing the expressions for $\Delta^{m}$ and $\Delta^{n}$, we obtain that (whether sellers multihome or singlehome under both organizations) a seller's willingness to invest is larger (provided all other sellers invest the same) if trade occurs via strategic platform rather than via free platforms if the investment leads to a larger increase of the buyers' net surplus than of the sellers' net surplus. We indicated above that platform size and thus the number of multi- and singlehoming sellers depend on the type of platform organization. Consider the case that each seller joins at least one platform if platforms are strategic, i.e. $n_{s}^{i *} \geq 1 / 2$ (which is equivalent to $\left.u+\pi>2 C_{s}+2 \tau_{s}\right)$. Then even more sellers multi-home if platforms are public. However, if in both types of platform organization sellers who single-home are decisive to determine the individual contribution to investment (which is assumed to be the same for each seller), then we obtain the following result with respect to sellers' incentives. ${ }^{14}$

Proposition 2 Suppose $n_{s}^{i *} \geq 1 / 2$ and sellers who singlehome determine investment levels. In the competitive bottleneck model in which sellers are on the multihoming side, strategic trading platforms give stronger incentives for sellers to innovate if the change of the buyers' surplus is larger than the change of the sellers' surplus, i.e.

$$
\Delta^{m}>\Delta^{n} \Longleftrightarrow u\left(y^{\prime}\right)-u(y)>\pi\left(y^{\prime}\right)-\pi(y)
$$

Note that this condition is more demanding than the corresponding rule in the singlehoming environment, rule (10), provided that profits are increasing in the investment level $y$. This is due to the fact that the sellers' surplus can be better extracted by the strategic platform when the sellers can multihome.

\footnotetext{
${ }^{14}$ The sellers located in $\left[1-\pi /\left(2 \tau_{s}\right), 1-v_{s}^{*} / \tau_{s}\right]$ and in $\left[\pi /\left(2 \tau_{s}\right), v_{s}^{*} / \tau_{s}\right]$ multihome if platforms are public, but singlehome if platforms are strategic. For them, the condition for incentives to innovate to be higher under intermediated trade is more stringent: $\Delta^{m}>\Delta^{n} \Longleftrightarrow u\left(y^{\prime}\right)-u(y)>3\left[\pi\left(y^{\prime}\right)-\pi(y)\right]$.
} 
If $n_{s}^{i *}<1 / 2$, we cannot translate a seller's willingness to pay for the innovation oneto-one into total sellers investments because in this case the equilibrium number of active sellers depends on the type of platform organization (and only active sellers contribute to the joint investment). Since more sellers are active under public platforms, the condition that strategic platforms lead to more investment than public platforms tends to become even more demanding. ${ }^{15}$ We do not consider this case any further.

\section{Competitive bottlenecks when buyers multihome}

We analyze the same model as in the previous section with the only difference that the role of buyers and sellers is reversed, that is, sellers singlehome and buyers multihome. ${ }^{16}$ Hence, for given investment levels only the buyer and seller indices have to be reversed and the analysis of the previous section applies. Rewriting expression (14), we have that the equilibrium net surplus of sellers (gross of transportation cost) is now equal to:

$$
v_{s}^{*}=\frac{1}{4 \tau_{b}}\left(u^{2}+4 \pi u+\pi^{2}-2(u+\pi) C_{b}\right)-\tau_{s}-C_{s}
$$

On the other hand, if trade takes place via two free public platforms, the surplus of sellers is obtained from expression (15):

$$
v_{s}^{n}=\frac{1}{2 \tau_{b}} \pi u .
$$

Again we compare the sellers' incentives to innovate under the two organizations of the trading platforms. If buyers are on the multihoming side, we can show that trade via strategic platforms lead to stronger investment incentives than via free platforms if the joint surplus of buyers and sellers increases, which has to be the case under any potentially welfare-improving increase of the investment level.

\footnotetext{
${ }^{15} \mathrm{An}$ additional problem is that the timing of events has to be reinterpreted since the number of active sellers depends on the pricing by the platform and so should the total investment. However, according to the game, as presented in Section 2, investment decisions are taken in the first period. A possibility is to reinterpret the decision in stage 1 as a conditional promise, i.e. a commitment to invest a certain amount conditional on the decision to participate, which is taken at a later stage. This in turn implies that the participation decision in stage 3 will depend on the investment level that was agreed upon at stage 1.

${ }^{16}$ For instance, every Sunday morning, there are two flea markets in Brussels; their locations are sufficiently close for consumers to be able to visit both on the same morning; however, sellers are not mobile and stay put on a single market. This situation also applies to cases in which sellers sign exclusivity contracts with platforms but where buyers multi-home.
} 
Proposition 3 In the competitive bottleneck model in which sellers are on the singlehoming side, strategic trading platforms give stronger incentives for sellers to innovate if the joint buyers' and sellers' surplus increases, i.e.

$$
\Delta^{m}>\Delta^{n} \Longleftrightarrow u\left(y^{\prime}\right)-u(y)+\pi\left(y^{\prime}\right)-\pi(y)>0 .
$$

Proof. Incentives to innovate are computed as: $\Delta^{m}=v_{s}^{*}\left(y^{\prime}\right)-v_{s}^{*}(y)$ and $\Delta^{n}=v_{s}^{n}\left(y^{\prime}\right)-v_{s}^{n}(y)$. Using the above expressions for $v_{s}^{*}$ and $v_{s}^{n}$, developing and simplifying, we find

$$
\Delta^{m}-\Delta^{n}=\frac{1}{4 \tau_{b}}\left(u\left(y^{\prime}\right)-u(y)+\pi\left(y^{\prime}\right)-\pi(y)\right)\left(u\left(y^{\prime}\right)+u(y)+\pi\left(y^{\prime}\right)+\pi(y)-2 C_{b}\right) .
$$

As the second bracketed term is positive (otherwise, strategic platforms would not be able to make a profit), we check condition (17).

Note that this condition is less demanding than rule (10). The intuition is that the membership fee on the singlehoming side is substantially lower since platforms compete more fiercely for the singlehoming side.

\section{Applications}

In line with most of the literature on two-sided markets, we did not provide so far a microfoundation of $u$ and $\pi$. To fill the gap, we use a simple model according to which each seller offers an independent product, i.e. a product that is neither a substitute nor a complement for the other products. Each consumer has independent variable demand for each of the products. Suppose that inverse demand for each product is given by $P(q)=1-q^{\alpha}$ for $\alpha>0$. If $\alpha=1$, demand is linear; if $0<\alpha<1$, demand is convex, and if $\alpha>1$, demand is concave. Suppose that marginal cost of production is constant and equal to $0 \leq c<1$.

We consider three specific types of investments: sellers can invest in $R \& D$ in order to (i) reduce their marginal cost of production, (ii) improve the quality of their product, or (iii) enhance their ability to price discriminate. For each type of investment, we examine whether incentives to invest are higher under intermediated or non-intermediated trade. To facilitate the analysis, it is useful to summarize the results of Propositions 1 to 3 : incentives to innovate are higher under intermediated trade if and only if

- $u\left(y^{\prime}\right)-u(y)>0$ (both sides singlehome),

- $u\left(y^{\prime}\right)-u(y)>\pi\left(y^{\prime}\right)-\pi(y)$ (buyers singlehome, sellers can multihome),

- $u\left(y^{\prime}\right)-u(y)+\pi\left(y^{\prime}\right)-\pi(y)>0$ (sellers singlehome, buyers can multihome). 


\subsection{Cost reducing $R \& D$}

Each sellers decision problem at the last stage reduces to the simple monopoly maximization problem $\max _{q} \pi=\left(1-c-q^{\alpha}\right) q$. The profit-maximizing quantity is computed as:

$$
q=\left(\frac{1-c}{\alpha+1}\right)^{\frac{1}{\alpha}}
$$

We can derive the firm's profit and the consumer surplus at the profit-maximizing quantity:

$$
\begin{aligned}
& \pi(c)=\frac{\alpha(1-c)}{\alpha+1}\left(\frac{1-c}{\alpha+1}\right)^{\frac{1}{\alpha}}, \\
& u(c)=\frac{\alpha(1-c)}{(\alpha+1)^{2}}\left(\frac{1-c}{\alpha+1}\right)^{\frac{1}{\alpha}}=\frac{1}{\alpha+1} \pi(c) .
\end{aligned}
$$

Suppose there exists a process innovation that allows sellers to decrease the marginal cost of production from $c$ to $c^{\prime}$, with $0<c^{\prime} \leq c$. Both the firm's profit and the consumer surplus increase as a result of the cost reduction; that is, $u\left(c^{\prime}\right)-u(c)>0$ and $\pi\left(c^{\prime}\right)-\pi(c)>0$. It follows that intermediated trade provides higher incentives to invest in cost-reduction when both sides singlehome (from Proposition 1) and when sellers singlehome while buyers can multihome (from Proposition 3). As for the situation where buyers singlehome and sellers can multihome, incentives are higher under intermediated trade if and only if (from Proposition $2)$ :

$$
u\left(c^{\prime}\right)-u(c)>\pi\left(c^{\prime}\right)-\pi(c) \Longleftrightarrow \frac{1}{\alpha+1}\left(\pi\left(c^{\prime}\right)-\pi(c)\right)>\pi\left(c^{\prime}\right)-\pi(c),
$$

which is never true as, by assumption, $\alpha>0$. It follows that in the case where buyers singlehome and sellers can multihome, incentives to invest in cost-reduction are lower under intermediated trade.

\subsection{Quality improving R\&D}

By simply relabeling variables, we can replicate the analysis for a type of quality improving R\&D. Suppose that there exists a product innovation that shifts the inverse demand curve outward; namely, consider quality $s \geq 0$ with $P(q)=1+s-q^{\alpha}$. Marginal costs are here set equal to zero. Then, the profit maximization problem of each monopoly seller becomes: $\max _{q} \pi=\left(1+s-q^{\alpha}\right) q$, which is made equivalent to the above analysis by simply substituting $-c$ for $s$. The results of the previous subsection therefore carry over. 


\subsection{Investment in price discrimination}

The third type of investment we consider consists in joint data collection activities and in information sharing agreements among sellers. This investment allows sellers to practice some form of price discrimination and capture thereby a share $0 \leq \beta \leq 1$ of the consumer's surplus at a given price $p$. We can think of each seller setting a two-part tariff of the form: $T(p, q)=\beta C S(p)+p q$, where $C S(p)$ is the consumer's surplus at price $p$. A few lines of computation establish that

$$
C S(p)=\frac{\alpha}{\alpha+1}(1-p)^{\frac{\alpha+1}{\alpha}} .
$$

The innovation allows sellers to capture a larger share of the consumer's surplus (i.e., to increase $\beta$ ). For a given value of $\beta$, the seller chooses $p$ so as to maximize (to ease the computations, we set again the marginal cost to zero):

$$
\max _{p} \pi(p, \beta)=p(1-p)^{\frac{1}{\alpha}}+\beta \frac{\alpha}{\alpha+1}(1-p)^{\frac{\alpha+1}{\alpha}} .
$$

The profit-maximizing price is easily found as

$$
p^{*}(\beta)=\frac{\alpha(1-\beta)}{1+\alpha(1-\beta)}
$$

We check that $p^{*}(0)=\alpha /(1+\alpha)$ (profit-maximizing uniform price) and $p^{*}(1)=0$ (the variable part of the tariff is equal to the marginal cost in case of perfect price discrimination). We compute now the net gain from trade for each seller and for each buyer respectively as:

$$
\begin{aligned}
& \pi(\beta)=\pi\left(p^{*}(\beta), \beta\right)=\beta C S\left(p^{*}(\beta)\right)+p^{*}(\beta)\left(1-p^{*}(\beta)\right)^{\frac{1}{\alpha}} \\
& =\frac{\alpha}{\alpha+1}\left(\frac{1}{1+\alpha(1-\beta)}\right)^{\frac{1}{\alpha}} \\
& u(\beta)=(1-\beta) C S\left(p^{*}(\beta)\right)=(1-\beta) \frac{\alpha}{\alpha+1}\left(\frac{1}{1+\alpha(1-\beta)}\right)^{\frac{\alpha+1}{\alpha}} .
\end{aligned}
$$

We observe that $\pi(\beta)$ increases and $u(\beta)$ decreases with $\beta$ :

$$
\frac{d}{d \beta} u(\beta)=-\frac{\beta \alpha}{\alpha+1}\left(\frac{1}{1+\alpha(1-\beta)}\right)^{\frac{2 \alpha+1}{\alpha}}<0 .
$$

Therefore, $u\left(\beta^{\prime}\right)-u(\beta)<0<\pi\left(\beta^{\prime}\right)-\pi(\beta)$, which implies, from Propositions 1 and 2 , that intermediated trade provides lower incentives to innovate in price discrimination when both 
sides singlehome and when buyers singlehome while sellers can multihome. As for the third case (sellers singlehome, buyers can multihome), we observe that total surplus increases with $\beta$ :

$$
\begin{aligned}
\pi(\beta)+u(\beta) & =\frac{\alpha}{\alpha+1}\left(\frac{1}{1+\alpha(1-\beta)}\right)^{\frac{1}{\alpha}}\left(\frac{2-\beta+\alpha(1-\beta)}{1+\alpha(1-\beta)}\right) \\
\frac{d}{d \beta}(\pi(\beta)+u(\beta)) & =\frac{\alpha(1-\beta)}{(1+\alpha(1-\beta))^{2}}\left(\frac{1}{1+\alpha-\alpha \beta}\right)^{\frac{1}{\alpha}}>0
\end{aligned}
$$

Applying Proposition 3, we thus have that here intermediated trade provides higher incentives to innovate.

\subsection{Summary}

We collect the results of the three applications in the following proposition.

Proposition 4 Whether incentives to innovate are stronger under intermediated or nonintermediated trade depends on which side of the market singlehomes and on the nature of the innovation, as depicted in the following table (where "+" stands for stronger seller investment incentives if platforms are strategic):

\begin{tabular}{cccc}
\hline \hline & cost & quality & price \\
& reduction & improvement & discrimination \\
\hline \hline both sides singlehome & + & + & - \\
\hline sellers multihome & - & - & - \\
buyers singlehome & & & + \\
\hline sellers singlehome & + & + & + \\
buyers multihome & + & + & \\
\hline
\end{tabular}

\section{Conclusion}

In this paper, we analyze whether and how the fact that products are not sold on free, public, platforms but on competing, for-profit, platforms affects sellers' investment incentives. Investments in cost reduction, quality, or marketing measures are here the joint and coordinated efforts by sellers (in the form of horizontal arrangements). In particular, they may take the form of $R \& D$ cooperatives. We show that, in general, strategic intermediation is not neutral to such investment incentives. 
We build a model with many manufacturers and consumers and two competing intermediaries who charge membership or access fees on both sides of the market. We compare this situation to an environment in which manufacturers and consumers have free access to platforms. Clearly, the presence of strategic intermediaries reduces the rents that are available in the market. Therefore, one might suspect that sellers have weaker investment incentives with competing for-profit platforms. However, this is not necessarily the case. The reason is that investment incentives affect the size of the network effects and thus competition between intermediaries.

In particular, we show that results on the strength of investment incentives depends on which side of the market singlehomes and on the nature of the $R \& D$ cooperation. For instance, if both sides singlehome, incentives to invest in cost reduction are stronger with competing for-profit platforms, whereas incentives to invest in consumer targeting (that, e.g., improve the possibility of price discrimination) are weaker.

Our formal analysis suffers from a number of limitations that future research should endeavor to address. First, our analysis only allows for membership or participation fees and platforms do not charge for usage. In many real-world examples, platform also charge for usage on at least one side of the market (e.g., video game platforms receive royalties from game developers for each game sold). Unfortunately, a meaningful analysis of platforms which charge for both usage and membership is involved and we have preferred, in this paper, to concentrate on a simple framework. Also largely unexplored dynamic issues are likely to affect the choice of price strategies. This cannot be addressed in an atemporal pricing model.

Second, we have assumed that investments are the result of sellers's coordinated efforts. Naturally, it would be interesting to redo the present analysis under the assumption that investment decisions are, instead, uncoordinated. Finally, the scope of our analysis is also limited by our assumption that sellers take their pricing decisions independently of one another. In the appendix, we show that our results carry over to a situation of imperfect competition among sellers captured by a negative direct external effect among sellers. This can be interpreted as a congestion effect on the platform. In our specification, sellers' profits decrease linearly with the number of sellers present on a platform, but, as we show, pricing decisions remain independent. A more general approach would be to consider strategic interaction in pricing decisions (e.g., that sellers produce imperfectly differentiated products). However, such interaction makes the platform choice game much more complex to solve than in the present setting. We leave this issue for further research. 


\section{Appendix. Competition among sellers}

We introduce competition on the sellers' side in the following simple way: we now express the seller's surplus on platform $i$ as

$$
v_{s}^{i}=n_{b}^{i} \pi-\gamma n_{s}^{i}-M_{s}^{i},
$$

with $\gamma>0$. According to this formulation, each seller still earns $\pi$ per buyer he interacts with, but now looses $\gamma$ per seller present on his platform. We can think of some form of congestion on the platform to explain the latter effect. It follows that, all other things equal, a seller prefers the platform visited by the lowest number of sellers. In that sense, sellers are competing with one another for platform access.

We show here that the results of Propositions 1 to 3 remain valid under this alternative specification.

Two-sided single-homing. The expressions for the numbers of buyers and sellers at the two platforms are now given by:

$$
\begin{aligned}
& n_{b}^{i}=\frac{1}{2}+\frac{\left(2 n_{s}^{i}-1\right) u-\left(M_{b}^{i}-M_{b}^{j}\right)}{2 \tau_{b}}, \\
& n_{s}^{i}=\frac{1}{2}+\frac{\left(2 n_{b}^{i}-1\right) \pi-\left(M_{s}^{i}-M_{s}^{j}\right)}{2\left(\tau_{s}+\gamma\right)} .
\end{aligned}
$$

Solving this system of linear equations we obtain

$$
\begin{aligned}
n_{b}^{i} & =\frac{1}{2}+\frac{u\left(M_{s}^{j}-M_{s}^{i}\right)+\left(\gamma+\tau_{s}\right)\left(M_{b}^{j}-M_{b}^{i}\right)}{2\left(\tau_{b}\left(\gamma+\tau_{s}\right)-u \pi\right)}, \\
n_{s}^{i} & =\frac{1}{2}+\frac{\pi\left(M_{b}^{j}-M_{b}^{i}\right)+\tau_{b}\left(M_{s}^{j}-M_{s}^{i}\right)}{2\left(\tau_{b}\left(\gamma+\tau_{s}\right)-u \pi\right)} .
\end{aligned}
$$

Comparing with expressions (7) and (8) obtained in the paper (with $\gamma=0$ ), we observe that the only difference is that $\left(\gamma+\tau_{s}\right)$ is substituted for $\tau_{s}$. Therefore, we obtain equilibrium membership fees from the previous ones by just making this substitution; that is:

$$
M_{s}^{*}=C_{s}+\gamma+\tau_{s}-u \text { and } M_{b}^{*}=C_{b}+\tau_{b}-\pi \text {. }
$$

It follows that at equilibrium, $n_{b}^{1 *}=n_{b}^{2 *}=\frac{1}{2}$ and $n_{s}^{1}=n_{s}^{2}=\frac{1}{2}$, so that the equilibrium net surplus of sellers and buyers (gross of transportation cost) are equal to:

$$
\begin{aligned}
v_{s}^{*} & =\frac{1}{2} \pi+u-\left(C_{s}+\tau_{s}\right)-\frac{3}{2} \gamma, \\
v_{b}^{*} & =\frac{1}{2} u+\pi-\left(C_{b}+\tau_{b}\right) .
\end{aligned}
$$


If interaction takes place on free public platforms, then it easily found (by setting $M_{s}^{j}=$ $M_{s}^{i}=M_{b}^{j}=M_{b}^{i}=0$ in the above expressions) that

$$
v_{s}^{i}=\frac{1}{2}(\pi-\gamma) \text { and } v_{b}^{i}=\frac{1}{2} u,
$$

Considering now the sellers' incentives to innovate, we observe that nothing changes with respect to the previous case as the competition effect comes as an additive term in the sellers' surplus functions. So, when computing incentives to innovate $\Delta^{n}$ and $\Delta^{m}$, the term in $\gamma$ disappears and we are left with the same expressions as before, implying that the result of Proposition 1 carries over.

Competitive bottlenecks when sellers multihome. It is easily checked that the equilibrium numbers of buyers and sellers on each platform can be derived from expressions (11) and (12) by simply substituting $\left(\gamma+\tau_{s}\right)$ for $\tau_{s}$ :

$$
\begin{aligned}
n_{b}^{i} & =\frac{1}{2}+\frac{u\left(M_{s}^{j}-M_{s}^{i}\right)+\left(\gamma+\tau_{s}\right)\left(M_{b}^{j}-M_{b}^{i}\right)}{2\left(\tau_{b}\left(\gamma+\tau_{s}\right)-u \pi\right)} \\
n_{s}^{i} & =\frac{\pi}{\left(\gamma+\tau_{s}\right)}\left(\frac{1}{2}+\frac{u\left(M_{s}^{j}-M_{s}^{i}\right)+\left(\gamma+\tau_{s}\right)\left(M_{b}^{j}-M_{b}^{i}\right)}{2\left(\tau_{b}\left(\gamma+\tau_{s}\right)-u \pi\right)}\right)-\frac{M_{s}^{i}}{\left(\gamma+\tau_{s}\right)} .
\end{aligned}
$$

Proceeding as in Section 4, we obtain

$$
\begin{aligned}
M_{b}^{1 *} & =M_{b}^{2 *}=\tau_{b}+C_{b}-\frac{\pi}{4\left(\gamma+\tau_{s}\right)}\left(3 u+\pi-2 C_{s}\right), \\
M_{s}^{1 *} & =M_{s}^{2 *}=\frac{1}{2} C_{s}+\frac{1}{4}(\pi-u), \\
n_{b}^{1 *} & =n_{b}^{2 *}=\frac{1}{2} \text { and } n_{s}^{1 *}=n_{s}^{2 *}=\frac{1}{4\left(\gamma+\tau_{s}\right)}\left(u+\pi-2 C_{s}\right), \\
v_{s}^{*} & =\frac{\tau_{s}}{4\left(\gamma+\tau_{s}\right)}\left(u+\pi-2 C_{s}\right), \\
v_{b}^{*} & =\frac{1}{4\left(\gamma+\tau_{s}\right)}\left(u^{2}+4 \pi u+\pi^{2}-2(u+\pi) C_{s}\right)-\tau_{b}-C_{b} .
\end{aligned}
$$

Repeating the analysis for interaction on free public platforms, we find:

$$
\begin{aligned}
& n_{b}^{1}=n_{b}^{2}=\frac{1}{2} \text { and } n_{s}^{1}=n_{s}^{2}=\frac{1}{2\left(\gamma+\tau_{s}\right)} \pi, \\
& v_{s}^{n}=\frac{\tau_{s}}{2\left(\gamma+\tau_{s}\right)} \pi \text { and } v_{b}^{n}=\frac{1}{2\left(\gamma+\tau_{s}\right)} \pi u .
\end{aligned}
$$

Considering sellers' incentives to innovate, we compute:

$$
\begin{aligned}
\Delta^{m} & = \begin{cases}\frac{\tau_{s}}{2\left(\gamma+\tau_{s}\right)}\left(u\left(y^{\prime}\right)-u(y)+\pi\left(y^{\prime}\right)-\pi(y)\right) & \text { (multihoming sellers) }, \\
\frac{\tau_{s}}{4\left(\gamma+\tau_{s}\right)}\left(u\left(y^{\prime}\right)-u(y)+\pi\left(y^{\prime}\right)-\pi(y)\right) & \text { (singlehoming sellers) }\end{cases} \\
\Delta^{n} & = \begin{cases}\frac{\tau_{s}}{\left(\gamma+\tau_{s}\right)}\left(\pi\left(y^{\prime}\right)-\pi(y)\right) & \text { (multihoming sellers) } \\
\frac{\tau_{s}}{2\left(\gamma+\tau_{s}\right)}\left(\pi\left(y^{\prime}\right)-\pi(y)\right) & \text { (singlehoming sellers) }\end{cases}
\end{aligned}
$$


Comparing the expressions for $\Delta^{m}$ and $\Delta^{n}$ (focusing on sellers who multihome or singlehome in the two cases), we have that $\Delta^{m}>\Delta^{n}$

$$
\begin{aligned}
& \Longleftrightarrow \quad \frac{\tau_{s}}{2\left(\gamma+\tau_{s}\right)}\left(u\left(y^{\prime}\right)-u(y)+\pi\left(y^{\prime}\right)-\pi(y)\right)>\frac{\tau_{s}}{\left(\gamma+\tau_{s}\right)}\left(\pi\left(y^{\prime}\right)-\pi(y)\right) \\
& \Longleftrightarrow u\left(y^{\prime}\right)-u(y)>\pi\left(y^{\prime}\right)-\pi(y),
\end{aligned}
$$

which is the same condition as in Proposition 2.

Competitive bottlenecks when buyers multihome. We proceed as in Section 5 and find the following expressions under the new specification:

$$
\begin{aligned}
n_{s}^{i} & =\frac{1}{2}+\frac{\pi\left(M_{b}^{j}-M_{b}^{i}\right)+\tau_{b}\left(M_{s}^{j}-M_{s}^{i}\right)}{2\left(\tau_{b}\left(\gamma+\tau_{s}\right)-u \pi\right)}, \\
n_{b}^{i} & =\frac{u}{\tau_{b}}\left(\frac{1}{2}+\frac{\pi\left(M_{b}^{j}-M_{b}^{i}\right)+\tau_{b}\left(M_{s}^{j}-M_{s}^{i}\right)}{2\left(\tau_{b}\left(\gamma+\tau_{s}\right)-u \pi\right)}\right)-\frac{M_{b}^{i}}{\tau_{b}}, \\
M_{s}^{1 *} & =M_{s}^{2 *}=\gamma+\tau_{s}+C_{s}-\frac{u}{4 \tau_{b}}\left(3 \pi+u-2 C_{b}\right), \\
M_{b}^{1 *} & =M_{b}^{2 *}=\frac{1}{2} C_{b}+\frac{1}{4}(u-\pi), \\
n_{s}^{1 *} & =n_{s}^{2 *}=\frac{1}{2} \text { and } n_{b}^{1 *}=n_{b}^{2 *}=\frac{1}{4 \tau_{b}}\left(u+\pi-2 C_{b}\right), \\
v_{s}^{*} & =\frac{1}{4 \tau_{b}}\left(u^{2}+4 \pi u+\pi^{2}-2(\pi+u) C_{b}-2 \gamma \tau_{b}\right)-\left(C_{s}+\tau_{s}+\gamma\right), \\
v_{b}^{*} & =\frac{1}{4}\left(u+\pi-2 C_{b}\right) .
\end{aligned}
$$

If interaction takes place on free public platform, we have:

$$
\begin{aligned}
& n_{s}^{1}=n_{s}^{2}=\frac{1}{2} \text { and } n_{b}^{1}=n_{b}^{2}=\frac{1}{2 \tau_{b}} u \\
& v_{s}^{n}=\frac{1}{2 \tau_{b}} u \pi-\gamma \frac{1}{2} \text { and } v_{b}^{n}=\frac{1}{2} u .
\end{aligned}
$$

Writing $\left(u_{0}, \pi_{0}\right)$ for $(u(y), \pi(y))$ and $\left(u_{1}, \pi_{1}\right)$ for $\left(u\left(y^{\prime}\right), \pi\left(y^{\prime}\right)\right)$, we compute

$$
\begin{aligned}
\Delta^{m} & =\frac{1}{4 \tau_{b}}\left(u_{1}^{2}+4 \pi_{1} u_{1}+\pi_{1}^{2}-2\left(\pi_{1}+u_{1}\right) C_{b}-\left(u_{0}^{2}+4 \pi_{0} u_{0}+\pi_{0}^{2}-2\left(\pi_{0}+u_{0}\right) C_{b}\right)\right), \\
\Delta^{n} & =\frac{1}{2 \tau_{b}}\left(u_{1} \pi_{1}-u_{0} \pi_{0}\right) .
\end{aligned}
$$

It follows that

$$
\Delta^{m}-\Delta^{n}=\frac{1}{4 \tau_{b}}\left(u_{1}+\pi_{0}+\pi_{1}+u_{0}-2 C_{b}\right)\left(u_{1}-u_{0}-\pi_{0}+\pi_{1}\right),
$$

which is positive as long as $u_{1}+\pi_{1}>u_{0}+\pi_{0}$ or $u\left(y^{\prime}\right)-u(y)+\pi\left(y^{\prime}\right)-\pi(y)>0$, i.e., which is the same condition as in Proposition 3. 


\section{References}

[1] Armstrong, M. (2006), Competition in Two-Sided Markets, Rand Journal of Economics, forthcoming.

[2] Boudreau, K. (2006), Does Opening a Platform Generate More Innovation? An Empirical Study, MIT Sloan Working Paper 4611-06.

[3] Caillaud, B., and Jullien, B. (2003). Chicken and Egg: Competition among Intermediation Service Providers, Rand Journal of Economics 34, 521-552.

[4] Clements, M. and Hiroshi, O. (2005), Indirect Network Effects an the Product Cycle: Video Games in the U.S., 1994-2002, Journal of Industrial Economics 53, 515-542

[5] Evans, D. (2003), Some Empirical Aspects of Multi-sided Platform Industries, Review of Network Economics 2, 191-209

[6] Evans, D. and Schmalensee, R. (2005), The Industrial Organization of Markets with Two-Sided Platforms, mimeo.

[7] Gehrig, T. (1993), Intermediation in Search Markets, Journal of Economics and Management Strategy 2, 97-120.

[8] Kaiser, U. and Wright, J. (2006), Price Structure in Two-sided Markets: Evidence from the Magazine Industry, International Journal of Industrial Organization 24, 1-28.

[9] Kamien, M.I., E. Muller and I. Zang (1992), Research Joint Ventures and R\&D Cartels), American Economic Review 82, 1293-1306.

[10] Lerner, J. (2002), Did Microsoft Deter Software Innovation? mimeo.

[11] Rochet, J-C. and Tirole, J. (2003), Platform Competition in Two-Sided Markets, Journal of the European Economic Association 1, 990-1024.

[12] Rochet, J-C. and Tirole, J. (2006), Two-Sided Markets: A Progress Report, Rand Journal of Economics, forthcoming.

[13] Rust, J. and Hall, G. (2003), Middlemen versus Market Makers: A Theory of Competitive Exchange, Journal of Political Economy 111, 353-403. 
[14] Spulber, D. (1999), Market Microstructure: Intermediaries and the Theory of the Firm, New York: Cambridge University Press.

[15] Spulber, D. (2003), Market Microstructure and Incentives to Invest, Journal of Political Economy 110, 352-381.

[16] Stennek, J. (2006), Exclusive Quality, mimeo. 
Département des Sciences Économiques de l'Université catholique de Louvain

Institut de Recherches Économiques et Sociales

Place Montesquieu, 3

1348 Louvain-la-Neuve, Belgique 